\title{
The Topology of Belief, Belief Revision and Defeasible Knowledge
}

\author{
Alexandru Baltag ${ }^{1}$, Nick Bezhanishvili $^{2}$, Aybüke Özgün ${ }^{1}$, Sonja Smets $^{1 \star}$ \\ 1 University of Amsterdam, The Netherlands \\ 2 Utrecht University, The Netherlands
}

\begin{abstract}
We present a new topological semantics for doxastic logic, in which the belief modality is interpreted as the closure of the interior operator. We show that this semantics validates Stalnaker's epistemicdoxastic axioms [23], and indeed it is the most general (extensional) semantics validating them. We prove, among other things, that in this semantics the doxastic logic KD45 is sound and complete with respect to the class of all extremally disconnected topological spaces. We also give a topological semantics for conditional belief and show its connection to the operation of updating with "hard information" (modeled by restricting the topology to a subspace). We show that our topological notions fit well with the defeasibility analysis of knowledge: topological knowledge coincides with undefeated true belief. We compare our semantics to the older topological interpretation of belief in terms of Cantor derivative (Steinsvold 2006), arguing in favor of our new semantics.
\end{abstract}

\section{Introduction}

Ever since Edmund Gettier published his famous counterexamples [14], formal epistemologists have been concerned with understanding the relation between belief and knowledge, and in particular with finding the conditions that distinguish an item of belief (no matter how true and justified) from an item of knowledge. This question can be approached from two sides: 1) we start with the weakest notion of true justified (or justifiable) belief and add conditions in order to argue that they establish a "good" (e.g. factive, correctly-justified, unrevisable, coherent, stable, truth-sensitive) notion of knowledge; or 2) we start from a chosen notion of knowledge and weaken it to obtain a "good" (e.g. consistent, introspective, possibly false) notion of belief. Most research in formal epistemology follows the first approach. In particular, the standard topological semantics for knowledge (in terms of the interior operator) can be included within this first approach, as based on a notion of knowledge as "correctly justified belief": according to the interior semantics, a proposition (set of possible worlds) $P$ is known if there exists some "true evidence" (i.e. an open set $A$ containing the real world $s$ ) that entails $P$ (i.e. $A \subseteq P$ ). Another example of the first approach is

\footnotetext{
* S. Smets contribution to this paper has received funding from the ERC under the European Community's 7th Framework Programme/ERC Grant agreement no. 283963.
} 
the so-called defeasibility analysis of knowledge proposed by Lehrer and Paxson [18], Klein [16] and other authors: knowledge is defined as undefeated (justified) true belief; i.e. true belief that cannot be defeated by any new (true) evidence.

While most research in formal epistemology follows the first approach, the second approach has to date received much less attention from formal logicians. This is rather surprising, since such a "knowledge-first" approach has been persuasively defended by one of most influential contemporary epistemologists (Williamson [27]). The only formal account following this second approach that we are aware of (prior to our own work) is the one given by Stalnaker [23], using a relational semantics for knowledge, based on Kripke models in which the accessibility relation is a directed preorder. In this setting, Stalnaker argues that the "true" logic of knowledge is the modal logic $S 4.2$ and that belief can be defined as the epistemic possibility of knowledge. In other words, believing $p$ is equivalent to "not knowing that you don't know" p:

$$
B p=\neg K \neg K p .
$$

Stalnaker justifies this identity from first principles based on a particular notion of belief, namely belief as "subjective certainty". Stalnaker refers to this concept as "strong belief", but we prefer to call it full belief ${ }^{3}$. What is important about this type of belief is that it is subjectively indistinguishable from knowledge: an agent "fully believes" $p$ iff in fact she "believes that she knows" $p$.

The resulting conception of knowledge is clearly different from Williamson's (who rejects the KK principle), but it is closely related to the above-mentioned defeasibility analysis [17]. Indeed, Stalnaker proceeds to formalize AGM belief revision, based on a special case of the above semantics, in which the accessibility relation is assumed to be a weakly connected preorder, and (conditional) beliefs are defined by minimization. This validates the AGM principles for belief revision. Stalnaker shows that in this special case his notion of knowledge coincides with (a simplified and idealized version of) Lehrer's concept of undefeated (justified) true belief: i.e. true belief that cannot be defeated by (revising with) any new (true) evidence. However, this special case supports a stronger logic of knowledge (the system S4.3). Since Stalnaker defends the weaker $S 4.2$ as the "true" logic of knowledge, he is lead to argue against the defeasibility theory.

In this paper, we aim to generalize Stalnaker's formalization, making it independent from the concept of plausibility order and from relational semantics. In fact, we are looking for the most general (extensional) 4 semantics for full belief (in the above-mentioned sense), validating Stalnaker's principles for epistemicdoxastic logic. We prove that the most general such semantics is a topological

\footnotetext{
${ }^{3}$ We adopt this terminology both because we want to avoid the clash with the very different notion of strong belief (due to Battigalli and Siniscalchi [2]) that is standard in epistemic game theory, and because we think that the intuitions behind Stalnaker's notion are very similar to the ones behind Van Fraassen's probabilistic concept of full belief [13].

4 An extensional semantics is one that assigns the same meaning to sentences having the same extension. Essentially, an extensional semantics takes the meaning of a sentence to be given by a "UCLA proposition": a set of possible worlds.
} 
one, that extends the standard topological interpretation of knowledge (as interior operator) with a new topological semantics for belief, given by the closure of the interior operator (with respect to an extremally disconnected topology). We compare our new semantics with the older topological interpretation of belief in terms of Cantor derivative, giving several arguments in favor of our semantics.

We prove that the logic of knowledge and belief with respect to our semantics is completely axiomatized by Stalnaker's epistemic-doxastic principles. Furthermore, we show that the complete logic of knowledge in this setting is indeed the system $S 4.2$, while the complete logic of belief is the standard system $K D 45$. We also formalize a notion of conditional belief $B^{P} Q$, using the relativization of the original topology to (the subspace corresponding to) the set $P$. Relativization itself is used to formalize the action of learning new (true) information. This allows us to model belief revision of a more general type than the one axiomatized by the AGM theory: the topological model validates only (appropriate versions of) the AGM axioms 1-6, but not necessarily the more controversial axioms 7 and 8 . We show that, in this more general setting, Stalnaker's objections to the defeasibility theory of knowledge do not apply: over arbitrary topological spaces, Stalnaker's notion of knowledge (having $S 4.2$ as its complete logic, and interpreted as topological interior) coincides with undefeated (justified) true belief.

\section{Background: Topological Interpretation of Knowledge}

\subsection{The Interior Semantics for Modal Logic}

We start by recalling the standard topological semantics of modal (epistemic) logic, originating in the work of Tarski and McKinsey [19]. We consider the standard unimodal language $\mathcal{L}_{K}$ with a countable set of propositional letters Prop, and a modal operator $K$. Formulas are defined as usual by

$$
\varphi::=\perp|p| \neg \varphi|\varphi \wedge \varphi| \varphi \vee \varphi \mid K \varphi,
$$

where $p \in$ Prop. Abbreviations for the connectives $\rightarrow$ and $\leftrightarrow$ are standard. The possibility operator $\langle K\rangle \varphi$ is defined as $\langle K\rangle \varphi:=\neg K \neg \varphi$.

For the basic definitions of general topology we refer to [11] or any other textbook in General Topology. A topological model $\mathcal{M}=(X, \tau, \nu)$ is a tuple where $(X, \tau)$ is a topological space and $\nu$ is a valuation, i.e., a map $\nu:$ Prop $\rightarrow$ $\mathcal{P}(X)$. We let $\mathrm{Cl}$ and Int denote the closure and interior operators, respectively.

Definition 1. Let $\mathcal{M}=(X, \tau, \nu)$ be a topological model. The semantics for modal formulas is defined by the following inductive definition, where $p$ is a propositional variable:

$$
\begin{array}{ll}
\llbracket \perp \rrbracket^{\mathcal{M}}=\emptyset, & \llbracket p \rrbracket^{\mathcal{M}}=\nu(p) \\
\llbracket \varphi \wedge \psi \rrbracket^{\mathcal{M}}=\llbracket \varphi \rrbracket^{\mathcal{M}} \cap \llbracket \psi \rrbracket^{\mathcal{M}} & \llbracket \varphi \vee \psi \rrbracket^{\mathcal{M}}=\llbracket \varphi \rrbracket^{\mathcal{M}} \cup \llbracket \psi \rrbracket^{\mathcal{M}} \\
\llbracket \neg \varphi \rrbracket^{\mathcal{M}}=X \backslash \llbracket \varphi \rrbracket^{\mathcal{M}} & \llbracket K \varphi \rrbracket^{\mathcal{M}}=\operatorname{Int} \llbracket \varphi \rrbracket^{\mathcal{M}}
\end{array}
$$

As $\langle K\rangle \varphi$ is equivalent to $\neg K \neg \varphi$, it is easy to see that $\llbracket\langle K\rangle \varphi \rrbracket^{\mathcal{M}}=\mathrm{Cl} \llbracket \varphi \rrbracket^{\mathcal{M}}$. 
We say that $\varphi$ is true in $\mathcal{M}=(X, \tau, \nu)$ if $\llbracket \varphi \rrbracket^{\mathcal{M}}=X$. We will skip the index $\mathcal{M}$ if it is clear from the context. We say that $\varphi$ is valid in $(X, \tau)$ if it is true in every model based on $(X, \tau)$. Soundness and completeness of modal logics wrt topological semantics is defined in the same way as for the Kripke semantics.

Proposition 1. (see e.g. [3], [20] and [8]) The modal logic S4 is sound and complete wrt all topological spaces.

\subsection{Epistemic Interpretation: open sets as pieces of evidence}

The original reason for interpreting interior as knowledge was that the Frechet axioms for interior match exactly the $S 4$ axioms for knowledge, and in particular the principles

$$
\text { (T) } K p \rightarrow p
$$

of Truthfulness of Knowledge ("factivity") and

$$
(K K) \quad K p \rightarrow K K p
$$

of Positive Introspection of Knowledge (known as axiom 4 in modal logic).

Philosophically, one of the best arguments in favor of the topological semantics is negative: namely, the fact that it does not validate the principle

$$
\neg K p \rightarrow K \neg K p
$$

This principle, known as (5) or Negative Introspection, is rejected by essentially all philosophers. One of its undesirable consequences is that it makes it impossible for a rational agent to have wrong beliefs about her knowledge: she always knows whatever she believes that she knows. This is known in the literature as Voorbrak's paradox [26]: it contradicts the day-to-day experience of encountering agents who believe they know things that they do not actually know ${ }^{5}$.

But, even beyond the issue of negative introspection, the topological semantics can arguably give us a deeper insight into the nature of knowledge and its evidential basis than the usual Kripke semantics. From an extensional point of view, the properties $U$ that are directly observable by an agent naturally form an open basis for a topology: closure under finite intersections captures an agent's ability to combine finitely many pieces of evidence into a single piece ${ }^{6}$. A proposition $P$ is true at world $w$ if $w \in P$. If an open $U$ is included in a set $P$, then we can say that proposition $P$ is entailed (supported, justified) by evidence $U$. Open neighbourhoods $U$ of the actual world $w$ play the role of sound (correct, truthful) evidence. The actual world $w$ is in the interior of $P$ iff there exists such a sound piece of evidence $U$ that supports $P$. So the agent "knows" $P$ if she

\footnotetext{
5 This common experience can be considered the starting point of all epistemological reflection, and historically played such a role, see e.g. in Platonic dialogues.

${ }^{6}$ But see van Benthem and Pacuit [5] for a more general logical account of evidencemanagement which relaxes this assumption: by using instead a neighbourhood semantics, this account can deal with agents who have not yet managed to combine all their pieces if evidence.
} 
has a correct justification for $P$ (based on a sound piece of evidence supporting $P)$. Moreover, open sets will then correspond to properties that are in principle verifiable by the agent: whenever they are true they can be known. Dually, closed sets will correspond to falsifiable properties. See Vickers [25] and Kelly [15] for more on this interpretation and its connections to Epistemology, Logic and Learning Theory.

So the knowledge-as-interior conception can be seen as an implementation of one of the most widespread intuitive responses to Gettier's challenge: knowledge is "correctly justified belief" (rather than being simply true justified belief). To qualify as knowledge, not only the content of one's belief has to be truthful, but its evidential justification has to be sound.

\subsection{Extensions and Improvements}

The interior-based semantics for knowledge has been extended to multiple agents [4], to common knowledge $[1,6]$, to logics of learning ("topo-logic", see [20]), to topological versions of dynamic-epistemic logic [28]. See [3] for a comprehensive survey of the field.

But there are two other topologically-based logics that are of particular interest in this paper. The first is an alternative semantics for modal logic, in terms of Cantor's derivative operation, which has been proposed as a semantics for belief. We will give a critical presentation of this alternative in Section 6. The second is a logic that strengthens $S 4$, namely:

$$
S 4.2=S 4+(\langle K\rangle K p \rightarrow K\langle K\rangle p) .
$$

By $L+\varphi$ we denote the smallest normal modal logic containing $L$ and $\varphi$.

Recall that a topological space $\langle X, \tau\rangle$ is called extremally disconnected if the closure of every open subset of $X$ is open.

Proposition 2. [3, p. 253] S4.2 is sound and complete wrt all extremally disconnected topological spaces.

We also recall that a topological space $(X, \tau)$ is called an Alexandroff space if the intersection of open sets of $X$ is open. It is well known that Alexandroff spaces correspond to reflexive and transitive Kripke frames, see e.g., [3], [20] or [8]. Moreover, the evaluation of modal formulas in an Alexandroff space coincides with their evaluation in the corresponding Kripke frame.

A Kripke frame $(X, R)$ is called directed if

$$
(\forall x, y, z)(x R y \wedge x R z) \rightarrow(\exists u)(y R u \wedge z R u)
$$

It is well known, see e.g., [9] or [10] that $S 4.2$ is sound and complete wrt reflexive, transitive and directed Kripke frames.

We give a few examples of extremally disconnected spaces. Alexandroff spaces corresponding to reflexive, transitive and directed Kripke frames are extremally disconnected. Another classical example of an extremally disconnected space is the Stone-Čech compactification $\beta(\mathbb{N})$ of the set of natural numbers with a discrete topology. Also it is well known that topological spaces that are Stonedual to complete Boolean algebras are extremally disconnected [22]. 


\section{The topology of full belief}

\subsection{Stalnaker's Epistemic-Doxastic Axioms}

In his paper [23], Stalnaker proposes a very interesting analysis of the relationship between knowledge and (justified or justifiable) belief. This is based on a conception of belief as "subjective certainty": from the point of the agent in question, her belief is subjectively indistinguishable from her knowledge. In this paper, we will refer to Stalnaker's notion as "full belief".

The bimodal language $\mathcal{L}_{K B}$ of knowledge and (full) belief is given recursively:

$$
\varphi::=\perp|p| \neg \varphi|\varphi \wedge \varphi| K \varphi \mid B \varphi,
$$

where $p \in$ Prop. We will also consider two unimodal fragments of this language $\mathcal{L}_{K}$ (having $K$ as its only modality) and $\mathcal{L}_{B}$ (having only $B$ ).

Stalnaker's epistemic-doxastic axioms for the logic $K B$ are given in the Table below.

\begin{tabular}{|c|c|l|}
\hline & Stalnaker's Epistemic-Doxastic Axioms & \\
\hline$(\mathrm{K})$ & $K(\varphi \rightarrow \psi) \rightarrow(K \varphi \rightarrow K \psi)$ & Knowledge is additive \\
$(\mathrm{T})$ & $K \varphi \rightarrow \varphi$ & Knowledge implies truth \\
$(\mathrm{KK})$ & $K \varphi \rightarrow K K \varphi$ & Positive introspection for $K$ \\
$(\mathrm{CB})$ & $B \varphi \rightarrow \neg B \neg \varphi$ & Consistency of belief \\
$(\mathrm{PI})$ & $B \varphi \rightarrow K B \varphi$ & (Strong) positive introspection of $B$ \\
$(\mathrm{NI})$ & $\neg B \varphi \rightarrow K \neg B \varphi$ & (Strong) negative introspection of $B$ \\
$(\mathrm{~KB})$ & $K \varphi \rightarrow B \varphi$ & Knowledge implies Belief \\
$(\mathrm{FB})$ & $B \varphi \rightarrow B K \varphi$ & Full Belief \\
\hline & Inference Rules & \\
\hline$(\mathrm{MP})$ & From $\varphi$ and $\varphi \rightarrow \psi$ infer $\psi$. & Modus Ponens \\
$(K-\mathrm{Nec})$ & From $\varphi$ infer $K \varphi$. & Necessitation \\
\hline
\end{tabular}

We will refer to this axiomatic system as $K B$. The axioms seem very natural and uncontroversial: the first three are the $S 4$ axioms for knowledge; $(\mathrm{CB})$ captures the consistency of beliefs, and in the context of the other axioms will be equivalent to the modal axiom (D) for beliefs: $\neg B \perp ;(\mathrm{PI})$ and (NI) capture strong versions of introspection of beliefs: the agent knows what she believes and what not; (KB) means that agents believe what they know; and finally, (FB) captures the essence of "full belief" as subjective certainty (the agent believes that she knows all the things that she believes). Finally, the rules of Modus Ponens and Necessitation seem uncontroversial (for implicit knowledge, if not for explicit knowledge) and are accepted by a majority of authors (and in particular, they are implicitly used by Stalnaker).

The above axioms imply that belief can be defined in terms of knowledge:

Proposition 3. (Stalnaker) The following equivalence is provable in the system $K B$ :

$$
B \varphi \leftrightarrow \neg K \neg K \varphi
$$

Moreover, all the axioms of the standard system KD45 for belief are provable in the system KB, and in particular: Kripke's axiom for belief $(B(\varphi \rightarrow \psi) \rightarrow$ 
$(B \varphi \rightarrow B \psi))$; the so-called axiom (D) $(\neg B \perp)$; axiom 4 (positive introspection) for belief $(B \varphi \rightarrow B B \varphi)$; the axiom 5 (negative introspection) for belief $(\neg B \varphi \rightarrow$ $B \neg B \varphi)$.

Finally, the formula $(\langle K\rangle K p \rightarrow K\langle K\rangle p)$ is also provable in $K B$ : i.e. all the axioms of the system $S 4.2$ hold for knowledge in the system $K B$.

\subsection{Our topological semantics for full belief}

Definition 2. An extensional (and compositional) semantics for the language $\mathcal{L}_{K B}$ of knowledge and full belief is a triplet $(X, B, K)$, where $X$ is a set of possible worlds, and $B: \mathcal{P}(X) \rightarrow \mathcal{P}(X)$ and $K: \mathcal{P}(X) \rightarrow \mathcal{P}(X)$ are unary operations on (sub)sets of worlds.

Any extensional semantics $(X, B, K)$, together with a valuation $\nu:$ Prop $\rightarrow$ $\mathcal{P}(X)$, gives us an extensional model $M=(X, B, K, \nu)$, in which we can interpret the formulas $\varphi$ of $\mathcal{L}_{K B}$ in the obvious way: the clauses for propositional connectives are the same as in the topological semantics above, and in rest we put

$$
\llbracket K \varphi \rrbracket^{\mathcal{M}}=K \llbracket \varphi \rrbracket^{\mathcal{M}} \quad \llbracket B \varphi \rrbracket^{\mathcal{M}}=B \llbracket \varphi \rrbracket^{\mathcal{M}} .
$$

As usual, a formula is valid in an extensional semantics $(X, B, K)$ if it is true at all worlds of all models $M=(X, B, K, \nu)$ based on it. An inference rule is valid if it preserves validity of formulas.

A special case of extensional semantics for the language $\mathcal{L}_{K B}$ is our proposed topological semantics:

Definition 3. A topological semantics for the language $\mathcal{L}_{K B}$ is an extensional semantics $\left(X, K^{\tau}, B^{\tau}\right)$, where $X$ is the domain of a topological space endowed with a topology $\tau, K^{\tau}=I^{\tau} t^{\tau}$ is the interior operator with respect to the topology $\tau$, and $B^{\tau}=\mathrm{Cl}^{\tau}\left(\operatorname{Int}^{\tau}\right)$ is the closure of the interior with respect to $\tau$.

Proposition 4. A topological space validates all the axioms and rules of the system KD45 for belief (with the semantics given above) iff it is extremally disconnected.

Proposition 5. A topological space validates all the axioms and rules of Stalnaker's system KB (with the semantics given above) iff it is extremally disconnected.

Now we can give a Topological Representation Theorem for extensional models of $K B$ :

Theorem 1. An extensional semantics $(X, B, K)$ validates all the axioms and rules of Stalnaker's system $K B$ iff it is a topological semantics given by an extremally disconnected topology $\tau$ on $X$ (such that $K=K^{\tau}=\operatorname{Int}^{\tau}$ and $B=$ $\left.B^{\tau}=\mathrm{Cl}^{\tau}\left(\operatorname{Int}^{\tau}\right)\right)$.

This last result shows that Stalnaker's axioms are just an alternative axiomatization of extremally disconnected topological spaces, in which both interior and the closure of interior are taken as primitive operations. The conclusion is that our topological semantics is indeed the most general (extensional compositional) semantics validating Stalnaker's axioms. 


\section{Conditioning, updates and defeasible knowledge}

Conditioning (with respect to some qualitative plausibility order or to a probability measure) is the most widespread way to model the learning of "hard" information ${ }^{7}$. The prior plausibility/probability assignment (encoding the agent's original beliefs before the learning) is changed to a new such assignment, obtained from the first one by conditioning with the new information $P$. In the qualitative case, this means just restricting the original order to $P$-worlds; while in the probabilistic case, restriction has to be followed by re-normalization (to ensure that the probabilities newly assigned to the remaining worlds add up to 1). In Dynamic Epistemic Logic, one makes also a distinction between simple ("static") conditioning and dynamic conditioning (also known as "update"). The first essentially corresponds to conditional beliefs: the change is made only locally, affecting only one occurrence of the belief operator $B \varphi$ (which is thus locally replaced by conditional belief $B^{P} \varphi$ ) or of the probability measure (which is locally replaced by conditional probability). In contrast, an update is a global change, at the level of the whole model (thus recursively affecting the meaning of all occurrences of the belief/probability operators). In this section, we investigate the natural topological analogues of static and dynamic conditioning.

Conditional beliefs. For every subset $P \subseteq X$ of a topological space $(X, \tau)$, we can generalize the belief operator in a natural way by relativizing the closure and interior operators to the set $P$ : we define the conditional belief operator $B^{P}: \mathcal{P}(X) \rightarrow \mathcal{P}(X)$ as

$$
B^{P} A=\mathrm{Cl}(P \cap \operatorname{Int}(P \rightarrow A)),
$$

where $P \rightarrow A:=(X \backslash P) \cup A$ is the set-theoretic version of material implication.

This immediately gives us a topological semantics for the language $\mathcal{L}_{K C B}$ obtained by adding to $\mathcal{L}_{K B}$ modalities $B^{\varphi} \psi$ for conditional belief. The additional semantic clause is

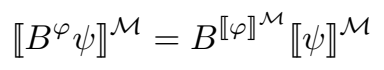

One possible justification of the above definition of conditional belief is that it validates an equivalence that generalizes the one for belief in a natural way:

Proposition 6. The following equivalences are valid in our semantics:

$$
B^{\varphi} \psi \longleftrightarrow \neg K(\varphi \rightarrow \neg K(\varphi \rightarrow \psi)) \longleftrightarrow \neg K \neg(\varphi \wedge K(\varphi \rightarrow \psi))
$$

This shows that (like simple beliefs) conditional belief can be defined in terms of knowledge. Unlike the case of simple belief, the converse is also true: knowledge can be defined in terms of conditional belief.

Proposition 7. The following equivalences are valid in our semantics:

$$
K \varphi \longleftrightarrow \neg B^{\neg \varphi} \top \longleftrightarrow \neg B^{\neg \varphi} \neg \varphi
$$

7 This term is used to denote information that comes with an inherent warranty of veracity, e.g. because of originating from an infallibly truthful source. 
The first of these equivalences shows that conditional belief is not a normal operator: it does not obey the Necessitation Rule, and in particular the formula $B^{\theta} \top$ is not in general a validity. The second equivalence above shows that in our theory the AGM Success Postulate has to be restricted to new inputs that are consistent with the agent's knowledge: $B^{\theta} \theta$ is not always valid, but $\neg K \neg \theta \rightarrow B^{\theta} \theta$ is valid. This restriction of Success is natural and desirable in the context of an epistemic logic.

Another axiom satisfied by our conditional beliefs is Revision Consistency:

Proposition 8. The formula $\neg B^{\varphi} \perp$ is valid in our topological semantics.

This is an analogue of the AGM postulate of Non-Vacuity. More generally, one can show that appropriate versions of the AGM Postulates 1-6 (with the abovementioned epistemic restriction of Success) can be stated in terms of conditional beliefs, and turn out to be valid in our semantics. However, AGM postulates 7 and 8 are not valid.

Updates. Another way to justify the above definition is by relating to the change of beliefs induced by learning (updating with) $P$. The natural topological analogue of dynamic conditioning (update) is the operation of taking the restriction (or "relativization") of a topology $\tau$ on $X$ to a subset $P \subseteq X$. What we obtain in this way is a subspace of the original topological space. Given a topological space $(X, \tau)$ and a set $P \subseteq X$, a space $\left(P, \tau_{P}\right)$ is called a subspace of $(X, \tau)$ if $\tau_{P}=\{U \cap P: U \in \tau\}$. It is well-known that the closure and interior operators in the relativized semantics $\left(P, \tau_{P}\right)$, denoted by $\mathrm{Cl}_{\tau_{P}}$ and $\operatorname{Int}_{\tau_{P}}$, respectively satisfy the equations for each $A \subseteq P$ :

$$
\begin{aligned}
& \mathrm{Cl}_{\tau_{P}}(A)=\mathrm{Cl}(A) \cap P, \\
& \operatorname{Int}_{\tau_{P}}(A)=\operatorname{Int}((X \backslash P) \cup A) \cap P .
\end{aligned}
$$

As a consequence, the belief operator in the relativized semantics $B^{\tau_{P}}$ relates to our conditional belief in the following way:

$$
B^{\tau_{P}} A=P \cap B^{P}(A \cap P)
$$

This motivates the topological belief semantics for conditional belief: to use J. van Benthem's expression, conditional beliefs "pre-encode" possible future belief changes. We can think of conditional beliefs as embodying the agent's belief revision policy: her strategy for belief change. The point is made even clearer if we introduce dynamic modalities for updates.

Update Modalities. We now add to the language new modalities $\langle! \varphi\rangle \psi$, to be thought of as (existential) dynamic modalities associated to updates: $\langle! \varphi\rangle \psi$ means that: $\varphi$ is true and after the agent learns this new true information, $\psi$ becomes true. The corresponding universal modality can be defined by duality: $[! \varphi] \psi=\neg\langle! \varphi\rangle \neg \psi$. Its meaning is that: if $\varphi$ is true then after the agent learns it, $\psi$ becomes true.

Semantics Let $\mathcal{M}=(X, \tau, \nu)$ be a topological model for $P \subseteq X$ we let $\mathcal{M}_{P}=\left(P, \tau_{P}, \nu_{P}\right)$ denote a new model, where $\left(P, \tau_{P}\right)$ is a subspace of $(X, \tau)$ 
and $\nu_{P}(p)=\nu(p) \cap P$, for each $p \in$ Prop. Given a formula $\varphi$ we will denote by $\mathcal{M}_{\varphi}$ the relativized model

$$
\mathcal{M}_{\varphi}=\left(\llbracket \varphi \rrbracket, \tau_{\llbracket \varphi \rrbracket}, \nu_{\llbracket \varphi \rrbracket}\right),
$$

where $\llbracket \varphi \rrbracket=\llbracket \varphi \rrbracket^{\mathcal{M}}$ is the interpretation of $\varphi$ in $\mathcal{M}$. The semantics of $\langle! \varphi\rangle \psi$ boils down now to evaluating $\psi$ in the relativized model:

$$
\llbracket\langle! \varphi\rangle \psi \rrbracket^{\mathcal{M}}=\llbracket \psi \rrbracket^{\mathcal{M}_{\varphi}} .
$$

The fact that conditional beliefs pre-encode belief updates is now captured by the following observation:

Proposition 9. Let $\mathcal{M}=(X, \tau, \nu)$ be a topological model, $\varphi$ be a formula and $p$ be an atomic sentence. Then we have

$$
\llbracket B p \rrbracket^{\mathcal{M}_{\varphi}}=\llbracket \varphi \wedge B^{\varphi} p \rrbracket^{\mathcal{M}}
$$

As a consequence, the following formula is valid:

$$
\langle! \varphi\rangle B p \longleftrightarrow\left(\varphi \wedge B^{\varphi} p\right) .
$$

More generally, the following Reduction Law for belief is valid, for arbitrary formulas $\varphi, \psi$ :

$$
\langle! \varphi\rangle B \psi \longleftrightarrow\left(\varphi \wedge B^{\varphi}\langle! \varphi\rangle \psi\right)
$$

Connection to the defeasibility theory of knowledge. As promised in the Introduction, we show now that in the generalized Belief Revision Theory given by our topological semantics for conditional beliefs, topological knowledge coincides with the one given by the defeasibility analysis.

Theorem 2. Let $\mathcal{M}=(X, \tau, \nu)$ be a topological model. The following are equivalent, for all worlds $x \in X$ and atomic sentences ${ }^{8} p$ :

1. $x \in \llbracket K p \rrbracket^{\mathcal{M}}$;

2. $x \in \llbracket \theta \rightarrow B^{\theta} p \rrbracket^{\mathcal{M}}$ for every formula $\theta$;

3. $x \in \llbracket[! \theta] B p \rrbracket^{\mathcal{M}}$ for every formula $\theta$;

4. $x \in \llbracket B p \rrbracket^{\mathcal{M}_{\theta}}$ for every formula $\theta$.

\section{Completeness Results}

Let us first look at the bimodal logic $K B$ of knowledge and (full) belief.

Theorem 3. The (sound and) complete logic of knowledge and belief on extremally disconnected spaces is given by Stalnaker's system KB.

\footnotetext{
8 The restriction to atomic sentences in the other clauses is necessary because of the so-called Moore sentence: these are epistemic formulas which change their truth value after being learnt.
} 
Next, we look at the unimodal fragment having $K$ as the only modality. In fact, this language has exactly the same expressivity as $K B$ (since the belief operator can be eliminated via the identity $B \varphi=\neg K \neg K \varphi)$. Moreover, we already know (by Proposition 2 in section 2.3) that the sound and complete logic of knowledge on extremally disconnected spaces is $S 4.2$.

Further, we look at the unimodal fragment having $B$ as the only modality: this logic is less expressive than the bimodal language $K B$, since knowledge is not reducible to belief. The completeness result for this logic, though unsurprising, is technically the hardest result in this paper (see proof in the Appendix):

Theorem 4. The complete logic of belief on extremally disconnected spaces is $K D 45$.

We now look at the logic $K C B$ of knowledge and conditional beliefs: this logic is as expressive as the logic $K B$ (and its fragment having $K$ as the only modality), since conditional beliefs can be defined from knowledge via the valid equivalence:

$$
B^{\varphi} \psi \leftrightarrow \neg K \neg(\varphi \wedge K(\varphi \rightarrow \psi))
$$

Note that (since knowledge and simple belief can also be defined in terms of conditional beliefs) the logic $K C B$ is also co-expressive with its fragment having only conditional belief modalities. As for completeness, this can be obtained trivially:

Theorem 5. The complete logic for the language $K C B$ can be obtained by putting together the system $S 4.2$ for the knowledge modality $K$ together with the above mentioned equivalence (that reduces conditional beliefs to knowledge) and the equivalence $B \varphi \leftrightarrow B^{\top} \varphi$ (reducing belief to conditional belief).

Finally, let us look at the corresponding dynamic logic.

Theorem 6. The logic obtained by adding update modalities to the language $K C B$ can be completely axiomatized by adding the following reduction axioms (from the above list) to any complete axiomatization of the logic $K C B$ :

$$
\begin{array}{ll}
\langle! \varphi\rangle p \leftrightarrow(\varphi \wedge p) & \langle! \varphi\rangle \neg \psi \leftrightarrow(\varphi \wedge\langle! \varphi\rangle \psi) \\
\langle! \varphi\rangle(\psi \wedge \theta) \leftrightarrow(\langle! \varphi\rangle \psi \wedge\langle! \varphi\rangle \theta) & \langle! \varphi\rangle K \psi \leftrightarrow(\varphi \wedge K\langle! \varphi\rangle \psi) \\
\langle! \varphi\rangle B^{\theta} \psi \leftrightarrow\left(\varphi \wedge B^{\langle! \varphi\rangle \theta}\langle! \varphi\rangle \psi\right) &
\end{array}
$$

\section{Comparison with related work}

We compare now our topological interpretation of belief with a different (and older) topological interpretation semantics that has been proposed for doxastic logic, using Cantor's derivative operator.

The Co-Derived Semantics for Belief Let $(X, \tau)$ be a topological space. We recall that a point $x$ is called a limit point (limit points are also called accumulation points) of a set $A \subseteq X$ if for each open neighbourhood $U$ of $x$ we have $(U \backslash\{x\}) \cap A \neq \emptyset$. Let $d(A)$ denote the set of all limit points of $A$. This set is called the derived set and $d$ is called the derived set operator. For each 
$A \subseteq X$ we let $t(A)=X \backslash d(X \backslash A)$. We call $t$ the co-derived set operator. Also recall that there is a close connection between the derived and co-derived set operators and the closure and interior operators. In particular, for each $A \subseteq X$ we have $\operatorname{Cl}(A)=A \cup d(A)$ and $\operatorname{Int}(A)=A \cap t(A)$. Unlike the closure operator there may exist elements of $A$ that are not its limit points. In other words, in general $A \not \subset d(A)$. Also note that for each $x \in X$ we have $x \notin d(x)$, where $d(x)$ is a shorthand for $d(\{x\})$.

We now define the semantics that we call co-derived set semantics for modal formulas. We again use $\langle B\rangle$ for $\neg B \neg$.

Definition 4. Let $\mathcal{M}=(X, \tau, \nu)$ be a topological model. We let the Booleans to be interpreted in the same way as in the interior-semantics. For each formula $\varphi$ we interpret the modal operators as follows:

$\llbracket B \varphi \rrbracket^{\mathcal{M}}=t\left(\llbracket \varphi \rrbracket^{\mathcal{M}}\right) \quad \llbracket\langle B\rangle \varphi \rrbracket^{\mathcal{M}}=d\left(\llbracket \varphi \rrbracket^{\mathcal{M}}\right)$.

We again skip the index $\mathcal{M}$ if it is clear from the context. SEE [3], [20] and $[8]$ for an overview of the results on the co-derived set semantics. Here we only mention that the logic $w K 4=K+((p \wedge B p) \rightarrow B B p)$ is complete wrt all topological spaces [12] and that the doxastic logic $K D 45$ is complete wrt so-called $D S O$-spaces, where a topological space $\langle X, \tau\rangle$ is called a $D S O$-space if it satisfies the $T_{D}$-separation axiom, ${ }^{9}$ for every $A \subseteq X$ the set $d(A)$ is open, and $(X, \tau)$ is dense-in-itself, i.e., $d(X)=X[24]$.

Criticism and comparison with our conception. Steinsvold [24] was the first to propose the co-derived set operator as a semantics for belief. However, this interpretation has a major disadvantage: it entails (not just the possibility, but) the necessity of error. To explain: 11 authors agree that one of the main characteristics of belief is the possibility of error: it is possible that some of the agent's beliefs are false. In other words, any good semantics for belief should allow for models and worlds at which some beliefs are false. However, we claim that, according to the co-derived semantics the existence of false beliefs is a necessary fact (holding for all possible agents at all possible worlds in all possible models!).

Indeed, as we pointed out above, for each $x \in X$ we always have $x \notin d(x)$. So $x \in B(X \backslash\{x\})$. Thus, at a point $x$ the agent believes $X \backslash\{x\}$, which is false (since $x \notin X \backslash\{x\})$. This means that in any topological model and any world in this model, there is at least one false belief. Hence, the co-derived set interpretation implies the "necessity of error": the actual world is always dis-believed.

We think this consequence is an intuitively undesirable property. It generally prevents any act of learning (updating with) the actual world. Indeed, the main problem of Formal Learning Theory (learning the true world, or the correct possibility, from a given set of possibilities) becomes automatically unattainable. Similarly, the physicist's dream of finding a true "theory of everything" is declared impossible by fiat, as a matter of logic. More importantly, even if necessity of error might seem realistic within a Lewisian "large-world interpretation" of possible-world semantics (in which each world must really come with a full

\footnotetext{
${ }^{9}$ Recall that the $T_{D}$ separation axiom states that every point is the intersection of a closed and open set. This condition is equivalent to $d(d(A)) \subseteq d(A)$, see e.g., [11].
} 
description of all the miriad of ontic facts of the world), this property seems completely unrealistic when we adopt the more down-to-earth "small-world" models that are common in Computer Science, Game theory and other applications. In these fields, the "worlds" in any usable model come only with the description of the facts that are relevant for the problem at hand: e.g. in a scenario involving the throwing of a fair coin, the relevant fact is the upper face of the coin. A model for this scenario will involve typically only two possible worlds: Head and Tail. Requiring that the agent must always have a false belief means in this context that the agent can never find out which of the coin's faces is the upper one: an obviously absurd conclusion!

We think that our topological semantics (which does not have the above defect) is better suited for doxastic logic than the co-derived semantics. Moreover, our proposal comes with an independent motivation: not only it is the "canonical" (most general) semantics for full belief (understood as subjective certainty), but it fits well with the standard interior-interpretation of knowledge, in the context of the defeasibility analysis: as we saw, in our semantics, undefeated-true-belief corresponds exactly to the interior operator.

Finally, here is an argument of a more technical nature. As mentioned above, the co-derived semantics validates the $K D 45$ axioms only on $D S O$-spaces, while our semantics validates them on extremally disconnected spaces. So the following result shows that our interpretation works on a larger class of models:

Proposition 10. Every DSO-space is extremally disconnected.

\section{Conclusions and future work}

From a philosophical perspective, the main importance of our paper is that it connects three different epistemological conceptions that were proposed as responses to Gettier's challenge: Stalnaker's epistemic definition of full belief (in the spirit of the "knowledge-first" approach), the "knowledge as correctlyjustified-belief" approach (underlying the topological semantics of knowledge) and the defeasibility analysis. We find it pleasing to see that, when correctly formalized (in full generality, without restricting to relational models or "rationally monotonic" revision), these three conceptions converge to the same thing.

We also find our topological semantics to be of independent philosophical interest and intrinsic intuitive value. Topologically, a point is in the interior of a set $\mathrm{P}$ iff it can be sharply distinguished (separated) from all non- $P$ points (by an open set); similarly. a point is in the closure of $P$ iff it is "very close" to $P$, i.e. it cannot be sharply distinguished from all $P$ points. Thus, an agent knows $P$ if she can sharply distinguish the actual world from all non- $P$-worlds; and the agent (fully) believes $P$ at a world $w$ if she cannot sharply distinguish $w$ from the worlds in which she has knowledge of $P$. Thus, one can say that belief is topologically "very close" to knowledge: indeed, the agent cannot sharply distinguish it from knowledge. We thus think that our topological semantics perfectly captures the essence of full belief as "subjective certainty". 


\section{References}

1. J. Barwise. Three views of common knowledge. In Proceedings of the second Conference on Theoretical Aspects of Reasoning about Knolwedge, pages 365-379, 1988.

2. P. Battigalli and M. Siniscalchi. Strong belief and forward induction reasoning. Journal of Economic Theory, 105:356-391, 2002.

3. J. van Benthem and G. Bezhanishvili. Modal logics of space. In Handbook of spatial logics, pages 217-298. Springer, Dordrecht, 2007.

4. J. van Benthem, G. Bezhanishvilli, B. ten Cate, and D. Sarenac. Modal logics for prudicts of topologies. Studia Logica, 84(3)(369-392), 2005.

5. J. van Benthem and E. Pacuit. Dynamic logics of evidence-based beliefs. Studia Logica, 99(1):61-92, 2011.

6. J. van Benthem and D. Sarenac. The geometry of knowledge. Aspects of universal Logic, 17, 2005.

7. N. Bezhanishvili. Pseudomonadic algebras as algebraic models of doxastic modal logic. Math. Log. Q., 48(4):624-636, 2002.

8. N. Bezhanishvili and W. van der Hoek. Structures for epistemic logic. In A. Baltag and S. Smets, editors, Logical and Informational Dynamics. A volume in honour of Johan van Benthem. Trends in Logic, Springer. To appear.

9. P. Blackburn, M. de Rijke, and Y. Venema. Modal Logic. Cambridge University Press, 2001.

10. A. Chagrov and M. Zakharyaschev. Modal Logic. The Clarendon Press, 1997.

11. R. Engelking. General topology, volume 6. Heldermann Verlag, Berlin, second edition, 1989.

12. L. Esakia. Weak transitivity-restitution. Study in logic, 8:244-254, 2001. In Russian.

13. B. van Fraassen. Fine-grained opinion, probability, and the logic of full belief. Journal of Philosophical logic, 24:349-377, 1995.

14. E. Gettier. Is justified true belief knowledge? Analysis, 23:121-123, 1963.

15. K. Kelly. The Logic of Reliable Inquiry. Oxford University Press, 1996.

16. P. Klein. A proposed definition of propositional knowledge. Journal of Philosophy, 68:471-482, 1971.

17. K. Lehrer. Theory of Knowledge. Westview Press, 2000.

18. K. Lehrer and T. Jr Paxson. Knowledge: Undefeated justified true belief. Journal of Philosophy, 66:225-237, 1969.

19. J. C. C. McKinsey and A. Tarski. The algebra of topology. Ann. of Math. (2), 45:141-191, 1944.

20. R. Parikh, L. Moss, and C. Steinsvold. Topology and epistemic logic. In Handbook of spatial logics, pages 299-341. Springer, Dordrecht, 2007.

21. K. Segerberg. An Essay in Classical Modal Logic. Philosophical Studies, Uppsala, 1971.

22. R. Sikorski. Boolean Algebras. Springer-Verlag, Berlin-Heidelberg-Newyork, 1964.

23. R. Stalnaker. On logics of knowledge and belief. Philosophical Studies, 128(1):169 199, 2006.

24. C. Steinsvold. Topological models of belief logics. PhD thesis, New York, NY, USA, 2006. AAI3283152.

25. S. Vickers. Topology via logic, volume 5 of Cambridge Tracts in Theoretical Computer Science. Cambridge University Press, Cambridge, 1989.

26. F.P.J.M. Voorbraak. As Far as I Know. PhD thesis, Utrecht University, 1993. 
27. T. Williamson. Knowledge and its Limits. Oxford Univ. Press, 2000.

28. J. Zvesper. Playing with Information. PhD thesis, University of Amsterdam ILLC PhD Thesis, 2010.

\section{Appendix}

We again denote $\neg B \neg$ by $\langle B\rangle$ and $\neg K \neg$ by $\langle K\rangle$.

We recall that if $\mathcal{M}=(X, \tau, \nu)$ is a topological model. Then for each formula $\varphi$ we interpret the modal operators as follows:

$$
\begin{aligned}
& \llbracket B \varphi \rrbracket^{\mathcal{M}}=\operatorname{Cl}\left(\operatorname{Int}\left(\llbracket \varphi \rrbracket^{\mathcal{M}}\right)\right), \\
& \llbracket\langle B\rangle \varphi \rrbracket^{\mathcal{M}}=\operatorname{Int}\left(\operatorname{Cl}\left(\llbracket \varphi \rrbracket^{\mathcal{M}}\right)\right) .
\end{aligned}
$$

We skip the indexes if they are clear from the context. The truth and validity of formulas and soundness and completeness of logics for the topological belief semantics is defined in the same way as for the co-derived set and interior semantics.

\section{A Proofs for Section 3}

\section{A.1 Proof of Proposition 4}

Now we will define a translation $(\cdot)^{*}$ from the formulas in the language $\mathcal{L}_{B}$ to the formulas in $\mathcal{L}_{K}$ using the following inductive definition:

$$
\begin{aligned}
& (\perp)^{*}=\perp, \\
& (p)^{*}=p, \text { where } p \in \text { Prop, } \\
& (\neg \varphi)^{*}=\neg \varphi^{*}, \\
& (\varphi \wedge \psi)^{*}=\varphi^{*} \wedge \psi^{*}, \\
& (B \varphi)^{*}=\langle K\rangle K \varphi^{*},
\end{aligned}
$$

It is useful to note that $(\langle B\rangle \varphi)^{*}=K\langle K\rangle \varphi^{*}$.

Lemma 1. Let $\mathcal{M}=(X, \tau, \nu)$ be a topological model. Then for each formula in the language $\mathcal{L}_{B}$ we have

$$
\llbracket \varphi \rrbracket^{\mathcal{M}}=\llbracket \varphi^{*} \rrbracket^{\mathcal{M}} .
$$

Proof. We prove the lemma by induction on the complexity of $\varphi$. The cases $\varphi=\perp, \varphi=p, \varphi=\neg \psi$ and $\varphi=\psi \wedge \chi$ are straightforward. Now let $\varphi=B \psi$, then

$$
\begin{aligned}
\llbracket \varphi \rrbracket^{\mathcal{M}} & \left.=\llbracket B \psi \rrbracket^{\mathcal{M}}=\mathrm{Cl}\left(\operatorname{Int}\left(\llbracket \psi \rrbracket^{\mathcal{M}}\right)\right) \quad \text { (by Ind. Hyp. }\right) \\
& =\mathrm{Cl}\left(\operatorname{Int}\left(\llbracket \psi^{*} \rrbracket^{\mathcal{M}}\right)\right)=\llbracket\langle K\rangle K \psi^{*} \rrbracket^{\mathcal{M}}=\llbracket(B \psi)^{*} \rrbracket^{\mathcal{M}}=\llbracket \varphi^{*} \rrbracket^{\mathcal{M}} .
\end{aligned}
$$

Lemma 2. Let $\mathcal{M}=(X, \tau, \nu)$ be a topological model based on an Alexandroff space $(X, \tau)$ and $\mathcal{M}^{\prime}=(X, R, \nu)$ be the corresponding reflexive and transitive Kripke model. Then

$$
\begin{array}{ll}
x \in \llbracket B \varphi \rrbracket^{\mathcal{M}} & \text { iff } \quad(\exists y)\left(x R y \wedge(\forall z)\left(y R z \rightarrow z \in \llbracket \varphi \rrbracket^{\mathcal{M}}\right)\right), \\
x \in \llbracket\langle B\rangle \varphi \rrbracket^{\mathcal{M}} & \text { iff } \quad(\forall y)\left(x R y \rightarrow(\exists z)\left(y R z \wedge z \in \llbracket \varphi \rrbracket^{\mathcal{M}}\right)\right) .
\end{array}
$$


Proof. By Lemma 1, $\llbracket B \varphi \rrbracket^{\mathcal{M}}=\llbracket\langle K\rangle K \varphi^{*} \rrbracket^{\mathcal{M}}$. As $\mathcal{M}$ is Alexandroff, $\llbracket\langle K\rangle K \varphi^{*} \rrbracket^{\mathcal{M}}=$ $\llbracket\langle K\rangle K \varphi^{*} \rrbracket^{\mathcal{M}^{\prime}}$. By $\llbracket \cdot \rrbracket^{\mathcal{M}^{\prime}}$ we denote the standard evaluation of a formula in a Kripke model $\mathcal{M}^{\prime}$. Spelling out the semantics of $\langle K\rangle$ and $K$ in a Kripke model, we obtain that $x \in \llbracket\langle K\rangle K \varphi^{*} \rrbracket^{\mathcal{M}^{\prime}}$ iff $(\exists y)\left(x R y \wedge(\forall z)\left(y R z \rightarrow z \in \llbracket \varphi^{*} \rrbracket^{\mathcal{M}^{\prime}}\right)\right)$. That $\mathcal{M}$ is Alexandroff together with Lemma 1 imply that the latter is equivalent to $(\exists y)\left(x R y \wedge(\forall z)\left(y R z \rightarrow z \in \llbracket \varphi \rrbracket^{\mathcal{M}}\right)\right)$. The case of $\langle B\rangle \varphi$ is similar.

Recall that

$$
K D 45=K+(\langle B\rangle \top)+(B p \rightarrow B B p)+(\langle B\rangle p \rightarrow B\langle B\rangle p) .
$$

Lemma 3. Let $\mathcal{M}=(X, \tau, \nu)$ be a topological model. Then for each $p \in$ Prop we have

1. $\llbracket\langle B\rangle \top \rrbracket=X$,

2. $\llbracket B p \rrbracket \subseteq \llbracket B B p \rrbracket$,

3. $\llbracket\langle B\rangle p \rrbracket \subseteq \llbracket B\langle B\rangle p \rrbracket$.

Proof. 1. By Lemma 1 we have

$$
\llbracket\langle B\rangle \top \rrbracket=\llbracket(\langle B\rangle \top)^{*} \rrbracket=\llbracket K\langle K\rangle \top \rrbracket=\operatorname{Int}(\mathrm{Cl}(\llbracket \top \rrbracket))=\operatorname{Int}(\mathrm{Cl}(X))=X
$$

2. By Lemma 1, $\llbracket B p \rrbracket=\llbracket(B p)^{*} \rrbracket=\llbracket\langle K\rangle K p \rrbracket$. We know that $\langle K\rangle K p \rightarrow\langle K\rangle K\langle K\rangle K p$ is true in $\mathcal{M}$ wrt the interior-semantics, i.e., we have $\llbracket\langle K\rangle K p \rightarrow\langle K\rangle K\langle K\rangle K p \rrbracket=$ $X$. Hence, $\llbracket B p \rrbracket=\llbracket\langle K\rangle K p \rrbracket \subseteq \llbracket\langle K\rangle K\langle K\rangle K p \rrbracket=\llbracket\langle K\rangle K\langle K\rangle K p^{*} \rrbracket=\llbracket B B p \rrbracket$.

3. As above, $\llbracket B p \rrbracket=\llbracket(B p)^{*} \rrbracket=\llbracket\langle K\rangle K p \rrbracket$. Moreover, $\llbracket B\langle B\rangle p \rrbracket=\llbracket(B\langle B\rangle p)^{*} \rrbracket=$ $\llbracket\langle K\rangle K K\langle K\rangle p \rrbracket=\llbracket\langle K\rangle K\langle K\rangle p \rrbracket$. Since $\llbracket K K\langle K\rangle p \rightarrow\langle K\rangle\langle K\rangle p \rrbracket=X$, we have $\llbracket K x\langle K\rangle p \rrbracket \subseteq \llbracket\langle K\rangle K\langle K\rangle p \rrbracket$, i.e., $\llbracket B p \rrbracket \subseteq \llbracket B\langle B\rangle p \rrbracket$.

It follows from Lemma 3 that the $D 45$ axioms are sound wrt the topological belief semantics. However, the $K$-axiom $B p \wedge B q \rightarrow B(p \wedge q)$ may not be sound wrt all topological models. This is why we need to restrict the class of our models to extremally disconnected spaces, that is, the models of $S 4.2$ discussed in the previous section.

\section{Lemma 4.}

$$
S 4+(\langle K\rangle K p \wedge\langle K\rangle K q \rightarrow\langle K\rangle K(p \wedge q))=S 4+(\langle K\rangle K p \rightarrow K\langle K\rangle p)=S 4.2 .
$$

Proof. We will use Kripke semantics. Suppose $\mathcal{M}=(X, R, \nu)$ is a reflexive, transitive and directed Kripke model. Let $x \in X$ be such that $x \in \llbracket\langle K\rangle K p \wedge$ $\langle K\rangle K q \rrbracket$. This means that $x \in \llbracket\langle K\rangle K p \rrbracket$ and $x \in \llbracket\langle K\rangle K q \rrbracket$. Hence, $\exists y, z \in X$ with $x R y$ and $x R z$ such that $y \in \llbracket K p \rrbracket$ and $z \in \llbracket K q \rrbracket$. By the directedness of $R$, there exists $w \in X$ such that $y R w$ and $z R w$. Since $y \in \llbracket K p \rrbracket, z \in \llbracket K q \rrbracket$ and $R$ is transitive, $w \in \llbracket K p \rrbracket$ and $w \in \llbracket K q \rrbracket$, i.e., $w \in \llbracket K p \wedge K q \rrbracket$. So $w \in \llbracket K(p \wedge q) \rrbracket$ and by transitivity, $x \in \llbracket\langle K\rangle K(p \wedge q) \rrbracket$.

For the other direction, it is easy to see that if $(X, R)$ is not directed, then $\langle K\rangle K p \wedge\langle K\rangle K q \rightarrow\langle K\rangle K(p \wedge q)$ is falsified in $\mathcal{M}$. 
Lemma 5. Let $(X, \tau)$ be a topological space. Then the $K$-axiom is valid in $X$ in the topological belief semantics iff $(X, \tau)$ is extremally disconnected.

Proof. Let $(X, \tau)$ be extremally disconnected and let $\mathcal{M}=(X, \tau, \nu)$ be a topological model based on $(X, \tau)$. Then $\mathcal{M}$ is a topological model of $S 4.2$ in the interior semantics. So by Lemma $4,\langle K\rangle K p \wedge\langle K\rangle K q \rightarrow\langle K\rangle K(p \wedge q)$ is true in $\mathcal{M}$ in the interior semantics. Thus, $\llbracket\langle K\rangle K p \wedge\langle K\rangle K q \rightarrow\langle K\rangle K(p \wedge q) \rrbracket^{\mathcal{M}}=X$. Note that $(B p \wedge B q \rightarrow B(p \wedge q))^{*}=\langle K\rangle K p \wedge\langle K\rangle K q \rightarrow\langle K\rangle K(p \wedge q)$. By Lemma 1 , we obtain that $\llbracket B p \wedge B q \rightarrow B(p \wedge q) \rrbracket^{\mathcal{M}}=X$. Therefore, the $K$-axiom is valid in $(X, \tau)$ in the topological belief semantics. The converse direction is similar.

We are now ready to prove

Proposition 4. A topological space validates all the axioms and rules of the system $K D 45$ (with the semantics given above) iff it is extremally disconnected.

Proof. The result follows immediately from Lemmas 3 and 5.

\section{A.2 Proof of Proposition 5}

Follows from Propositions 2 and 4.

\section{A.3 Proof of Theorem 1}

Proof. One direction is proved in the previous Proposition (Proposition 5 in Section 3.2), so let us look at the other direction. Suppose an extensional semantics $(X, B, K)$ validates the axioms and rules of $K B$. Then the validity of the $S 4$ axioms implies that $K$ satisfies the Frechet conditions for topological interior, and so it gives rise to a topology $\tau$ in which $K=\operatorname{Int}^{\tau}$. By the Proposition in the previous section, the $K B$ axioms imply that $B=\neg K \neg K$, i.e. $B=\neg \operatorname{Int}^{\tau} \neg \operatorname{Int}^{\tau}=\mathrm{Cl}^{\tau} \operatorname{Int}^{\tau}$. Hence, $(X, B, K)$ is a topological semantics, in the sense above, for a topology $\tau$. By the previous Proposition, the validity of $K B$ implies that $\tau$ is extremally disconnected.

\section{B Proofs for Section 4}

\section{B.1 Proof of Theorem 2}

Proof. We only prove the equivalence between the first two clauses, since the others follow trivially from this using the definition of relativized model and the semantics of dynamic modalities. For the left-to-right direction, assume $x \in$ $\llbracket K p \rrbracket^{\mathcal{M}}$. Then $x \in \operatorname{Int} \llbracket p \rrbracket^{\mathcal{M}} \subseteq \operatorname{Int}\left(\left(X \backslash \llbracket \theta \rrbracket^{\mathcal{M}}\right) \cup \llbracket p \rrbracket^{\mathcal{M}}\right)=\operatorname{Int}\left(\llbracket \theta \rrbracket^{\mathcal{M}} \rightarrow \llbracket p \rrbracket^{\mathcal{M}}\right)$. Assume now that $x \in \llbracket \theta \rrbracket^{\mathcal{M}}$. We have to show that $x \in \llbracket B^{\theta} p \rrbracket^{\mathcal{M}}$. For this, note that $x \in \llbracket \theta \rrbracket^{\mathcal{M}} \cap \operatorname{Int}\left(\llbracket \theta \rrbracket^{\mathcal{M}} \rightarrow \llbracket p \rrbracket^{\mathcal{M}}\right) \subseteq \operatorname{Cl}\left(\llbracket \theta \rrbracket^{\mathcal{M}} \cap \operatorname{Int}\left(\llbracket \theta \rrbracket^{\mathcal{M}} \rightarrow \llbracket p \rrbracket^{\mathcal{M}}\right)\right)=$

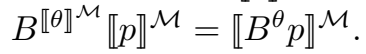


Conversely, assume that we have $x \in \llbracket \theta \rightarrow B^{\theta} p \rrbracket^{\mathcal{M}}$ for every formula $\theta$. Hence in particular, for $\theta:=\neg K p$, we have $x \in \llbracket \neg K p \rightarrow B^{K p} p \rrbracket^{\mathcal{M}}$. Assume now (towards a contradiction) $x \notin \llbracket K p \rrbracket^{\mathcal{M}}$, and hence $x \in \llbracket \neg K p \rrbracket^{\mathcal{M}}$. Putting these together by modus ponens, we obtain that $x \in \llbracket B^{K p} p \rrbracket^{\mathcal{M}}$. But this contradicts the following

Subclaim: The formula $B^{\neg K p} p$ is inconsistent.

Proof of Subclaim: We have the following sequence of deductions:

$B \neg K p p, \vdash \neg K \neg(\neg K p \wedge K(\neg K p \rightarrow p)) \vdash \neg K \neg(\neg K p \wedge K(\neg p \rightarrow K p) \vdash$ $\neg K \neg(\neg K p \wedge K(\neg p \rightarrow(\neg p \wedge K p))) \vdash \neg K \neg(\neg K p \wedge K(\neg p \rightarrow \perp)) \vdash \neg K \neg(\neg K p \wedge$ $K p) \vdash \neg K \neg \perp \vdash \neg K \top \vdash \neg \top \vdash \perp$.

This finishes the proof of the theorem.

\section{Proofs for Section 5}

\section{C.1 Proof of Theorem 3}

This follows trivially from our Topological Representation Theorem for extensional models of $K B$ (Theorem 1 in Section 3.2).

\section{C.2 Proof of Theorem 4}

For proving completeness we will need a few auxiliary definitions and lemmas. Let $(X, R)$ be a transitive frame. Recall that a non-empty subset $C \subseteq X$ is called a cluster if for each $x, y \in C$ we have $x R y$, and $(X, R)$ is called rooted if there exists $x \in X$ such that for each $y \in X$ we have $x R y$. We call $(X, R)$ cofinal if there exists a cluster $C \subseteq X$, which we call the cofinal cluster, such that for each $x \in X$ and each $y \in C$ we have $x R y$. We call a cofinal transitive frame a brush if $X \backslash C$, where $C$ is the cofinal cluster, is an irreflexive antichain i.e., for each $x, y \in X \backslash C$ we have $\neg(x R y)$. A brush, where $X \backslash C$ is a singleton set will be called a pin. If $\mathcal{M}$ is a Kripke model (not necessarily reflexive or transitive), then we assume that the semantics $\llbracket \varphi \rrbracket^{\mathcal{M}}$ is defined in a standard way.

The following theorem is well known see e.g., [10], [21], [7].

\section{Lemma 6.}

1. Each reflexive and transitive cofinal frame is an S4.2-frame. Moreover, S4.2 is sound and complete wrt finite rooted reflexive and transitive cofinal frames.

2. Each brush is a KD45-frame. Moreover, KD45 is sound and complete wrt finite brushes, indeed, wrt finite pins.

Let $(X, R)$ be a reflexive and transitive cofinal frame and let $C \subseteq X$ be the cofinal cluster. We define $R_{B}$ on $X$ by $x R_{B} y$ if $y \in C$ for each $x, y \in X$.

Lemma 7. Let $(X, R)$ be a reflexive and transitive cofinal frame with the cofinal cluster $C \subseteq X$. Then

1. $\left(X, R_{B}\right)$ is a brush. 
2. If $\nu$ is a valuation on $X, \mathcal{M}=(X, R, \nu)$ and $\mathcal{M}_{B}=\left(X, R_{B}, \nu\right)$, then for each formula $\varphi$ in the language $\mathcal{L}_{B}$ we have

$$
\llbracket \varphi \rrbracket^{\mathcal{M}}=\llbracket \varphi \rrbracket^{\mathcal{M}_{B}}
$$

Proof. (1) is easy to check.

We prove (2) by induction on the complexity of $\varphi$. The cases for $\varphi=\perp$, $\varphi=p, \varphi=\neg \psi$ and $\varphi=\psi \wedge \chi$ are straightforward. Now let $\varphi=B \psi$ and assume $x \in \llbracket \varphi \rrbracket^{\mathcal{M}}$. Then, by Corollary 2 , there exists $y \in X$ such that $x R y \wedge(\forall z)(y R z \rightarrow$ $\left.z \in \llbracket \psi \rrbracket^{\mathcal{M}}\right)$. Since $C$ is the cofinal cluster, we have $y R z$ for each $z \in C$. Hence, for every $z \in C$ we have $z \in \llbracket \psi \rrbracket^{\mathcal{M}}$. By the induction hypothesis, $\llbracket \psi \rrbracket^{\mathcal{M}}=\llbracket \psi \rrbracket^{\mathcal{M}_{B}}$. So, $C \subseteq \llbracket \psi \rrbracket^{\mathcal{M}_{B}}$. By the definition of $R_{B}$ we have that $x R_{B} u$ implies $u \in C$. Therefore, every $R_{B}$-successor of $x$ is in $\llbracket \psi \rrbracket^{\mathcal{M}_{B}}$. Thus, $x \in \llbracket B \psi \rrbracket^{\mathcal{M}_{B}}$.

Conversely, $x \in \llbracket B \psi \rrbracket^{\mathcal{M}_{B}}$. Then, by the definition of $R_{B}$, we have $C \subseteq$ $\llbracket \psi \rrbracket^{\mathcal{M}_{B}}$. By the induction hypothesis $\llbracket \psi \rrbracket^{\mathcal{M}}=\llbracket \psi \rrbracket^{\mathcal{M}_{B}}$. By Lemma $1, \llbracket \psi \rrbracket^{\mathcal{M}}=$

$\llbracket \psi^{*} \rrbracket^{\mathcal{M}}$. Since $C$ is the cofinal cluster in $(X, R)$, for every $y \in C$ we have $x R y$ and $y \in \llbracket K \psi^{*} \rrbracket^{\mathcal{M}}$. Therefore, $x \in \llbracket\langle K\rangle K \psi^{*} \rrbracket^{\mathcal{M}}=\llbracket(B \psi)^{*} \rrbracket^{\mathcal{M}}$. By Lemma 1 again we obtain that $x \in \llbracket B \psi \rrbracket^{\mathcal{M}}$.

For each Kripke frame $(X, R)$ we let $R^{+}$be the reflexive closure of $R$.

Lemma 8. Let $(X, S)$ be a brush. Then,

1. $\left(X, S^{+}\right)$is a reflexive and transitive cofinal frame.

2. If $\nu$ is a valuation on $X, \mathcal{M}=(X, S, \nu)$ and $\mathcal{M}^{+}=\left(X, S^{+}, \nu\right)$, then for each formula $\varphi$ in the language $\mathcal{L}_{B}$ we have

$$
\llbracket \varphi \rrbracket^{\mathcal{M}^{+}}=\llbracket \varphi \rrbracket^{\mathcal{M}}
$$

Proof. (1) is easy to check.

(2) It is easy to see that $\left(\mathcal{M}^{+}\right)_{B}=\mathcal{M}$. Then, by Lemma 7 we obtain that

$$
\llbracket \varphi \rrbracket^{\mathcal{M}^{+}}=\llbracket \varphi \rrbracket^{\left(\mathcal{M}^{+}\right)_{B}}=\llbracket \varphi \rrbracket^{\mathcal{M}} .
$$

Lemma 9. For each formula $\varphi$ in the language $\mathcal{L}_{B}$ we have

$$
S 4.2 \vdash \varphi^{*} \quad \text { iff } K D 45 \vdash \varphi .
$$

Proof. Suppose $S 4.2 \forall \varphi^{*}$. Then by Lemma $6(1)$, there exists a model $\mathcal{M}=$ $(X, R, \nu)$, where $(X, R)$ is a finite reflexive and transitive cofinal frame such that $\llbracket \varphi^{*} \rrbracket^{\mathcal{M}} \neq X$. By Lemma $1, \llbracket \varphi^{*} \rrbracket^{\mathcal{M}}=\llbracket \varphi \rrbracket^{\mathcal{M}}$. By Lemma $7, \mathcal{M}_{B}=\left(X, R_{B}, \nu\right)$ is a $K D 45$-model (moreover a brush) such that $\llbracket \varphi \rrbracket^{\mathcal{M}}=\llbracket \varphi \rrbracket^{\mathcal{M}_{B}} \neq X$, and by Lemma $6(2)$, we have $K D 45 \nvdash \varphi$.

Conversely, assume $K D 45 \forall \varphi$. by Lemma $6(2)$, there exists a model $\mathcal{M}=$ $(X, S, \nu)$, where $(X, S)$ is a finite pin such that $\llbracket \varphi \rrbracket^{\mathcal{M}} \neq X$. By Lemma $8, \mathcal{M}^{+}=$ $\left(X, R^{+}, \nu\right)$ is a finite reflexive and transitive cofinal frame and $\llbracket \varphi \rrbracket^{\mathcal{M}}=\llbracket \varphi \rrbracket^{\mathcal{M}^{+}}$. By Lemma $1, \llbracket \varphi \rrbracket^{\mathcal{M}^{+}}=\llbracket \varphi^{*} \rrbracket^{\mathcal{M}^{+}} \neq X$, and by Lemma $6(1)$, we have $S 4.2 \nvdash \varphi^{*}$. 
We are now ready to prove our main completeness result for the topological belief semantics.

Theorem 4. The complete logic of belief on extremally disconnected spaces is $K D 45$.

Proof. The soundness follows from Proposition 4. For completeness, assume that $K D 45 \forall \forall$. Then by Lemma $9, S 4.2 \forall \forall \varphi^{*}$. Thus, there exists a model $\mathcal{M}=(X, \tau, \nu)$ such that $(X, \tau)$ is extremally disconnected and $\llbracket \varphi^{*} \rrbracket^{\mathcal{M}} \neq X$. By Lemma 1 , we have $\llbracket \varphi \rrbracket^{\mathcal{M}}=\llbracket \varphi^{*} \rrbracket^{\mathcal{M}}$. So $\llbracket \varphi \rrbracket^{\mathcal{M}} \neq X$. Thus, we found an extremally disconnected space $(X, \tau)$ that refutes $\varphi$ in the topological belief semantics. This finishes the proof of the theorem.

Remark 1. Translation $(\cdot)^{*}$ can be extended to normal extensions of S4.2. In fact, we can show that the result of Lemma 9 holds for each normal modal logic $L$ such that $S 4.2 \subseteq L \subseteq S 4.2_{2}$, where $S 4.2_{2}$ is the logic obtained by adding to $S 4.2$ the axiom $b d_{2}[10$, p. 81$]$ not allowing in relational frames depth greater than 2 .

\section{C.3 Proofs of Theorems 5 and 6}

These proofs are by induction: applying the given reduction axioms recursively, one can eventually eliminate all the occurrences of belief and conditional belief (in Theorem 5) or all occurrences of dynamic modalities (in Theorem 6). Completeness of the axioms for the basic logic ( $S 4.2$ for knowledge in Theorem 5 , and axioms of $K C B$ in Theorem 6) takes care of the rest.

\section{Proofs for Section 6}

\section{D.1 Proof of Proposition 10}

Proof. Let $\mathcal{X}=(X, \tau)$ be a $D S O$-space and $U \in \tau$. Recall that for any $A \subseteq X$, $\mathrm{Cl}(A)=d(A) \cup A$. Hence, in particular, $\operatorname{Cl}(U)=d(U) \cup U$. As $\mathcal{X}$ being a $D S O$ space, $d(U)$ is an open subset of $X$. Thus, since $U$ is open as well, $d(U) \cup U=$ $\mathrm{Cl}(U)$ is open. 